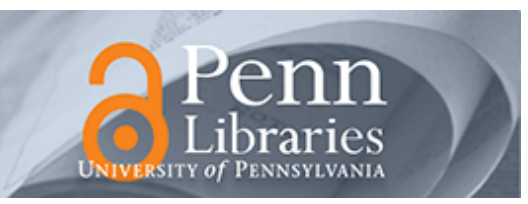

University of Pennsylvania

ScholarlyCommons

Departmental Papers (CIS)

Department of Computer \& Information Science

September 2007

\title{
Using Smart Cameras to Localize Self-Assembling Modular Robots
}

\author{
Babak Shirmohammadi \\ University of Pennsylvania, babaks@cis.upenn.edu \\ Camillo J. Taylor \\ University of Pennsylvania, cjtaylor@cis.upenn.edu \\ Mark Yim \\ University of Pennsylvania, yim@grasp.cis.upenn.edu \\ Jimmy Sastra \\ University of Pennsylvania \\ Mike Park \\ University of Pennsylvania \\ Follow this and additional works at: https://repository.upenn.edu/cis_papers
}

\section{Recommended Citation}

Babak Shirmohammadi, Camillo J. Taylor, Mark Yim, Jimmy Sastra, and Mike Park, "Using Smart Cameras to Localize Self-Assembling Modular Robots", . September 2007.

Copyright 2007 IEEE. Reprinted from Proceedings of the 1st ACM/IEEE International Conference on Distributed Smart Cameras, September 2007, pages 76-80.

This material is posted here with permission of the IEEE. Such permission of the IEEE does not in any way imply IEEE endorsement of any of the University of Pennsylvania's products or services. Internal or personal use of this material is permitted. However, permission to reprint/republish this material for advertising or promotional purposes or for creating new collective works for resale or redistribution must be obtained from the IEEE by writing to pubs-permissions@ieee.org. By choosing to view this document, you agree to all provisions of the copyright laws protecting it.

This paper is posted at ScholarlyCommons. https://repository.upenn.edu/cis_papers/386

For more information, please contact repository@pobox.upenn.edu. 


\title{
Using Smart Cameras to Localize Self-Assembling Modular Robots
}

\author{
Abstract \\ In order to realize the goal of self assembling or self reconfiguring modular robots the constituent \\ modules in the system need to be able to gauge their position and orientation with respect to each other. \\ This paper describes an approach to solving this localization problem by equipping each of the modules \\ in the ensemble with a smart camera system. The paper describes one implementation of this scheme on \\ a modular robotic system and discusses the results of a self assembly experiment.

\section{Keywords} \\ smart cameras, localization, modular robots, self-assembly

\section{Comments} \\ Copyright 2007 IEEE. Reprinted from Proceedings of the 1st ACM/IEEE International Conference on \\ Distributed Smart Cameras, September 2007, pages 76-80. \\ This material is posted here with permission of the IEEE. Such permission of the IEEE does not in any way \\ imply IEEE endorsement of any of the University of Pennsylvania's products or services. Internal or \\ personal use of this material is permitted. However, permission to reprint/republish this material for \\ advertising or promotional purposes or for creating new collective works for resale or redistribution must \\ be obtained from the IEEE by writing to pubs-permissions@ieee.org. By choosing to view this document, \\ you agree to all provisions of the copyright laws protecting it.
}




\section{USING SMART CAMERAS TO LOCALIZE SELF-ASSEMBLING MODULAR ROBOTS}

\author{
Babak Shirmohammadi, Camillo J. Taylor \\ University of Pennsylvania \\ Department of Computer and Information Science \\ \{babaks,cjtaylor\}@cis.upenn.edu
}

\author{
Mark Yim, Jimmy Sastra, Mike Park \\ University of Pennsylvania \\ Department of Mechanical Engineering \\ \{yim,jsastra,parkmich\}@ seas.upenn.edu
}

\begin{abstract}
In order to realize the goal of self assembling or self reconfiguring modular robots the constituent modules in the system need to be able to gauge their position and orientation with respect to each other. This paper describes an approach to solving this localization problem by equipping each of the modules in the ensemble with a smart camera system. The paper describes one implementation of this scheme on a modular robotic system and discusses the results of a self assembly experiment.
\end{abstract}

Index Terms - Smart Cameras, Localization, Modular Robots, Self Assembly

\section{INTRODUCTION}

As improvements in manufacturing continue to make sensors, actuators and processors smaller and cheaper, it has become increasingly appealing to think of constructing robotic systems out of collections of modular components. The underlying theme motivating research in the field of modular robotics is the idea that complex electromechanical systems can be assembled from collections of modules in the same way that our bodies are constructed from collections of cells. Such an arrangement offers a number of potential advantages. Firstly, constructing robots from a few basic modular pieces can reduce the cost of the system since the basic units can be mass produced much like Lego blocks. Secondly, modular systems can be more reliable and robust since the system could leverage the redundancy afforded by multiple active modules each having some actuation, sensing and computational capability. Finally, one of the most intriguing aspects of modular robots is the notion that the constituent modules of a robot could be reorganized or reconfigured depending on the dictates of the task at hand. Imagine, for instance, a robot that was originally configured as a humanoid that could reshape itself into a snake like form to worm through narrow passageways and then reconstitute its original form on the other side.

In order to realize this vision of self reconfiguring or self assembling modular robots, the individual modules in the system need to be able to gauge their position and orientation with respect to each other. This paper describes an approach to using self localizing smart camera systems to provide this positioning information.

In this paper we describe how self localization techniques originally developed for automatically localizing collections of distributed smart cameras [1] can be adapted to localize modular robotic components and, hence, to facilitate these types of self assembly operations. In the proposed scheme, each of the modular components is equipped with a smart camera system and a controllable light source. The modules use the lights to signal to each other and they determine their relative pose from the available image measurements.

There are several compelling advantages to using vision based methods for this localization task. Firstly, the required hardware, the imagers, computers and light sources are all amenable to miniaturization and are compatible with the manufacturing processes used to produce the robot modules themselves. Secondly, the proposed localization scheme requires relatively little power or communication. Thirdly the light beams used for localization are non-interfering which means that several nodes can self localize at the same time which is important in situations where tens or hundreds of modules need to operate simultaneously. Finally the imaging systems used for localization could also be used for other purposes such as sensing the environment to find obstacles or track targets. In fact, one could consider applications where modular robotic systems are used to automatically deploy camera systems to advantageous locations to provide better situational awareness.

The idea of using a team of robots as mobile landmarks for localization was proposed by Kurazume et al as a more accurate and robust alternative to robot positioning via dead reckoning $[2,3,4]$. The procedure was termed Cooperative Positioning (CP). With the proper sensor suites, the threedimensional configuration of a team of robots could be determined by sharing relative position and orientation information. Three different types of CP methods were outlined dependent upon the sensors and number of robots available.

Type 1 CP required only a pair of robots capable of measuring relative range, azimuth and elevation angles. At any given time, only one of the two robots would move, leaving the second to act as a landmark of known position. This 
scheme was later revisited by Rekleitis et al [5], and a planar version implemented using range and azimuth information obtained from a camera system.

Type 2 positioning required three robots capable of measuring relative azimuth and elevation angles with respect to a common axis. Based on these measurements it is possible to recover the configuration of the team up to a scale factor. If two of the robots are held stationary the third can be allowed to move with its position continuously updated via triangulation.

Type 3 positioning required three robots where relative angle and distance measurements could be made. This method allowed up to two of the three robots to move at any given time. Kurazume et al implemented and tested variants of this scheme using laser range finders. They showed far superior positioning results to those obtained from dead reckoning $[3,4]$. Grabowski et al [6] also implemented a planar version of a comparable scheme using the "Millibots" platforms. In this work radio frequency and ultrasonic emissions were combined to determine relative agent position and orientation for collaborative localization. Similair ideas for modular robot localization were explored by Zhang et al in [7]. In the commercial sector, IS Robotics (ISR) has also done similar work with their SWARM project. Using infra-red light, a team of robots is able to determine relative range and orientation information. The scheme described in this paper can be viewed as a form of cooperative localization where the relevant interrobot measurements are obtained using a distributed smart camera network.

The rest of the paper is organized as follows: Section 2 describes the implementation of the cooperative localization scheme on our modular robotic testbed. Section 3 describes our experience with an initial self assembly task which demonstrates the vision of a self organizing modular robotic system. Section 4 briefly discusses some areas of future work.

\section{IMPLEMENTATION}

The modular reconfigurable robot used in this work is based on the CKBot (Connector Kinetic roBot) design which is described more fully in [8]. The kinematics and connector strategy used in this design is typical of many chain style reconfigurable modular robots. Each module in the system consists of:

1. A laser cut plastic (ABS) body with a hobby servo actuator to control one rotational degree of freedom.

2. A controller (PIC18F2680) and associated hardware for implementing a Controller Area Network (CAN) and neighbor-to-neighbor IR communications protocol.

3. Four connector faces that pass the communications bus and power bus with an option of attaching at 90 rotations.

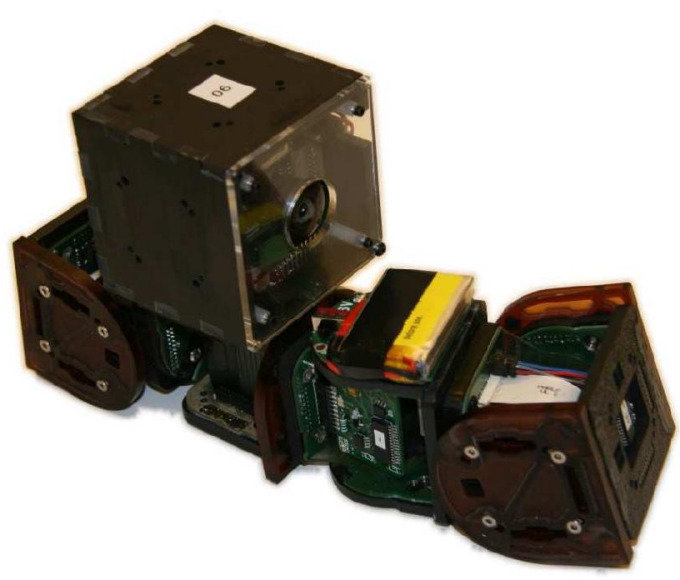

Fig. 1. Each cluster in the modular robot is composed of four CKBot modules and a smart camera system.

The individual modules can be connected to each other either using screws or through a set of magnetic linkages. These magnetic linkages are implemented using an array of rare earth magnets embedded in the connector faces of the modules. Four north facing and four south facing magnets arranged such that two opposing faces will attract each other. The magnets have enough strength hold a chain of seven modules together against gravity. These novel magnetic linkages enable modules in an assembly to dynamically attach or disconnect from each other.

For the reassembly experiment described in Section 3 the modules were grouped into 3 clusters each of which was composed of four CKbot modules and a smart camera module. The modules within each cluster were screwed together in a chain as shown in Figure 1 and the clusters were connected to each other via magnetic linkages. When the clusters are connected together they form a simple bipedal robot which can walk over flat terrain as depicted in Figure 3a; individually, the clusters can move about on the floor using snakelike gaits.

The smart camera modules used in this work are shown in Figure 2. Each of these camera modules contains a VGA imager outfitted with a fisheye lens, an Analog Devices Digital Signal Processor, a 3-axis accelerometer, and a wide-angle signaling LED which is used in the localization procedure. The smart camera module communicates with the other CKBot modules in the cluster via a CAN bus using the Robotics Bus protocol [9]. This CAN bus serves as the spinal cord of the cluster. Optionally, the smart camera modules can be outfitted with Bluetooth transceivers which enable wireless communication between camera modules. The smart cameras are powered by rechargeable lithium polymer batteries which are integrated into the design. 

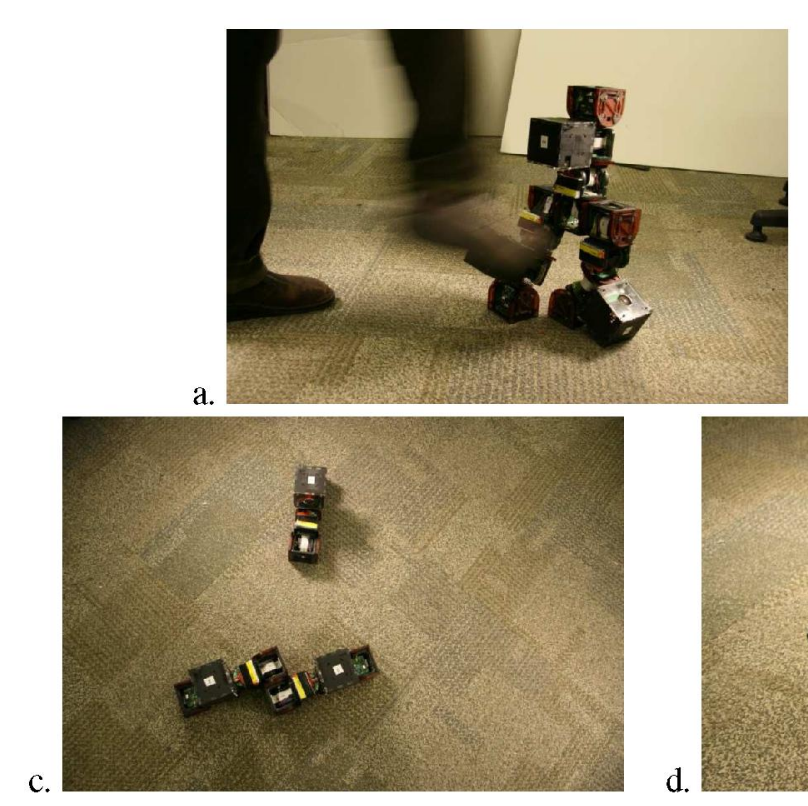

b.

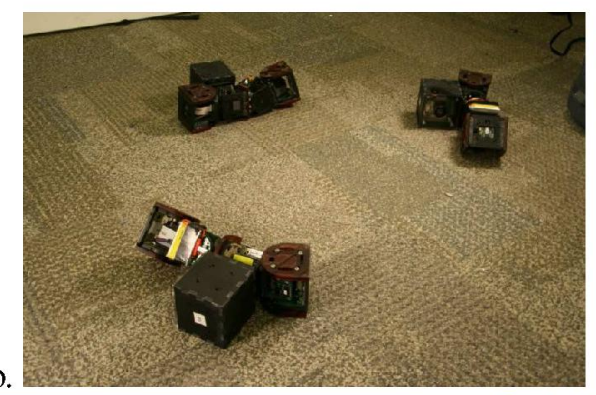

d.
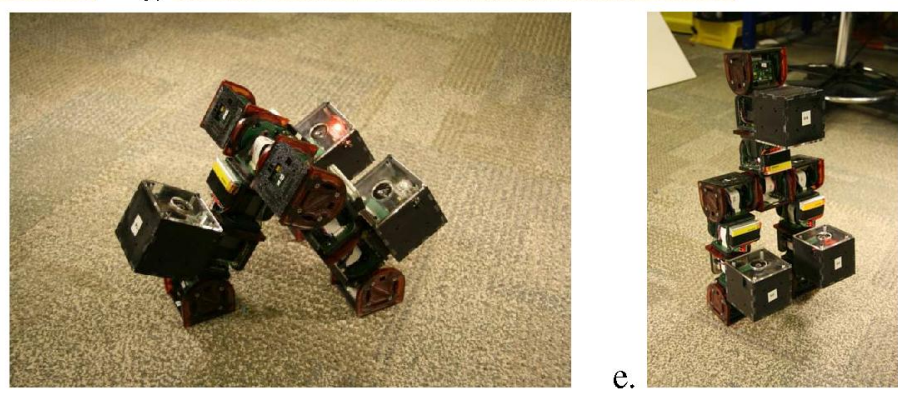

Fig. 3. This figure shows the phases in the automated reassembly experiment. Initially the modular robot is configured as a bipedal system. This system is rapidly disassembled into three pieces by a swift kick. The individual clusters than automatically reassemble themselves into the original configuration.

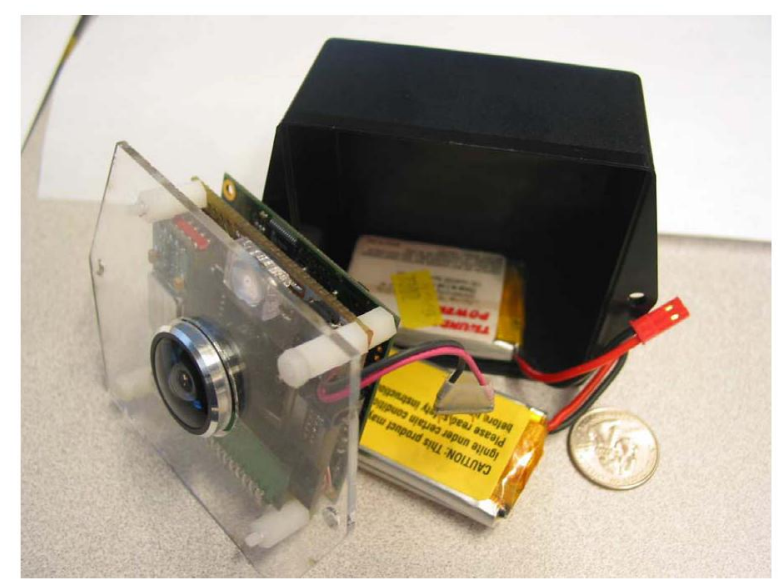

Fig. 2. Each smart camera module is equipped with an imager outfitted with a fisheye lens, a digital signal processor, an accelerometer, a CAN bus transciever, and an LED signalling light

\section{REASSEMBLY EXPERIMENT}

The goal of the reassembly experiment was to demonstrate, for the first time, a modular robotic system that was capable of self-reassembly after an explosive disassembly event. The sequence of events is described in Figure 3; Figure 3a shows the modular robot which is initially configured as a bipedal walking system. This robot is violently disassembled with a swift kick and breaks into its three constituent clusters which end up randomly distributed on the floor. The modules can determine that they are no longer connected by monitoring the status of inter cluster communication links. Each cluster then automatically orients itself with respect to the gravity so that the camera modules are on top using the measurements from the 3-axis accelerometers.

Once upright, the clusters perform a search to find each other visually. In our implementation, the camera nodes signal their presence by blinking their lights in a preset pattern. That is, each of the nodes would be assigned a unique string representing a fixed speed blink pattern such as 10110101 . The node would then turn its light on for 1 and off for 0 in the sequence prescribed by its string. These blink patterns provide a means for each of the nodes to locate other nodes in their images. They do this by collecting a sequence of images over time and analyzing the image intensity arrays to locate pixels whose intensity varies in an appropriate manner as described in [1].

This approach allows the camera node to both localize and uniquely identify neighboring nodes since the blink patterns 


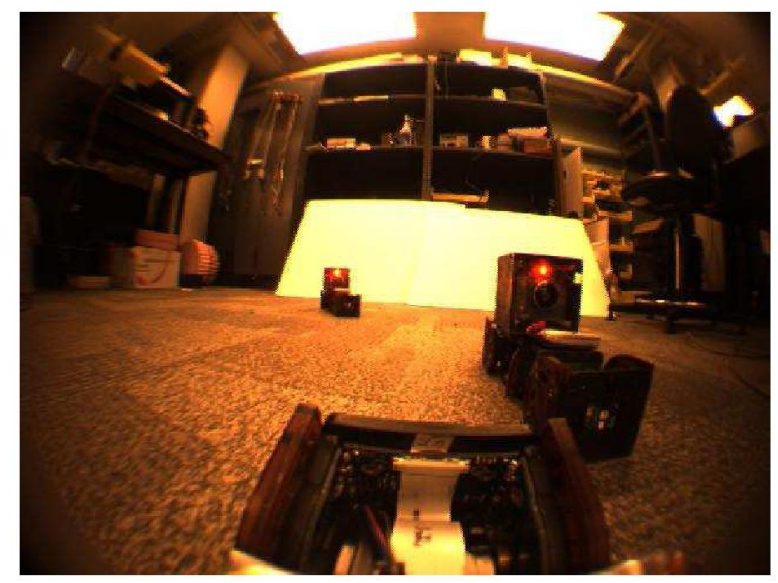

Fig. 4. This figure shows an image taken by one of the smart camera systems mounted on one of the clusters.

are individualized. In this scheme, each camera can determine the bearing to another as soon as there is a line of sight between two camera nodes. The size of the blinking light in the image is a function of relative angle and distance between the two camera nodes. Although this size is not a good indicator of range when the camera nodes are far apart, our experience shows it is a very effective measure at close range. This fits perfectly with docking where accurate measurements are only needed when the modules are close [10]. Figure 4 shows an image acquired by one of the camera modules during the docking procedure while Figure 5 shows how the size of the blinker in the image changes as a function of distance.

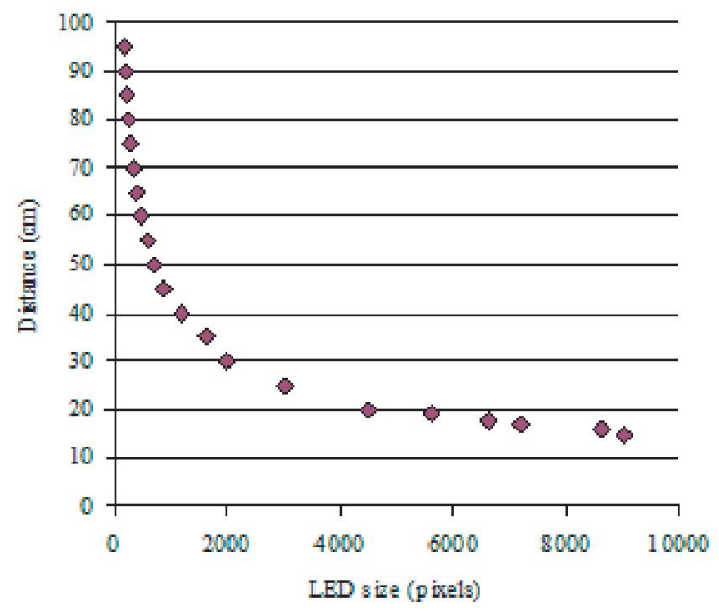

Fig. 5. This graph shows how the area of the blinker in pixels varies as a function of distance

In the first phase of the reassembly procedure the cluster corresponding to the torso and the cluster corresponding to the left leg rotate around in search of one another. When the left leg cluster locates the torso cluster it uses its estimate for the relative range and bearing to crawl towards that module and achieve the proper relative position for docking. Similarly, when the torso module sees the left leg it continually rotates around the vertical axis so that it maintains the correct relative orientation. In this manner the modules approach each other and orient themselves using a form of visual servoing. When the leg and torso clusters are sufficiently close the magnetic linkages reattach automatically and the communication and control links are reestablished.

Once the left leg and torso are docked, the torso module changes its blinker pattern. This serves as a signal for the other leg cluster to begin its approach and docking sequence. Here, the blinker systems are used not just for localization but also for signaling and control. The right leg docking proceeds in a similar manner to the left leg with the torso module rotating to achieve the expected relative orientation to the incoming leg.

Note that in this implementation the accelerometer subsystem is used to establish the orientation of the modules with respect to the gravity vector. This serves as a constraint which helps pairs of smart camera modules to determine their relative position and orientation from a pair of blinker sightings.

\section{FUTURE WORK}

In this work the distributed smart cameras provide a relative localization capability that can be used in a number of ways. To date, this capability has been used to demonstrate self-reassembly of a modular system but the same technology could be used to guide more purposeful reconfiguration operations where a modular robot may deliberately break into pieces so that it can reassemble itself in another form for a different purpose. We can imagine distributing a collection of modular components onto a planetary surface and having them automatically assemble themselves into structures that perform different functions. The fact that smart camera technology is amenable to miniaturization makes it possible to consider endowing each of the modules with the means to sense and localize other members of the ensemble.

Further, we envision using the camera systems mounted on the modules to sense the environment and provide information that the system can use to plan its activities. Like Argus, the hundred eyed shepherd of Greek mythology, we envision robotic systems that would continually capture imagery from multiple distributed vantage points and fuse this information to localize each other, to find and track moving objects and to build 3D representations of the scene. This information would allow the ensemble to plan its motions, to manipulate objects and to respond intelligently to changes in its environment. 


\section{CONCLUSION}

This paper describes a scheme for using embedded smart cameras to localize the elements of a reconfigurable modular robot. Experiments have been carried out which demonstrate that the system can be used to guide automated self-reassembly. The proposed scheme is well suited for use in modular robotic applications since the cameras, sensors and processors required are all amenable to miniaturization and are all compatible with the technologies used to manufacture the robot modules themselves.

The marriage of distributed smart cameras and modular robotics offers a number of compelling advantages. The cameras can provide relative positioning measurements which allow a set of distributed modules to act as a coordinated whole while the actuation and locomotion capabilities provided by the robotic modules can be used to deploy the smart cameras to advantageous viewpoints to support applications like 3D reconstruction and target tracking. We feel that this combination of technologies could offer a compelling approach to tackling a number of interesting applications.

\section{REFERENCES}

[1] Camillo J. Taylor and Babak Shirmohammadi, "Self localizing smart camera networks and their applications to 3d modeling," in Workshop on Distributed Smart Cameras (DSC 06), October 2006.

[2] R. Kurazume and S. Nagata, "Cooperative positioning with multiple robots," in IEEE International Conference on Robotics and Automation, 1994, pp. 1250-1257.

[3] R. Kurazume, S. Hirose, , S. Nagata, and N. Sashida, "Study on cooperative positioning system (basic principle and measurement experiment)," in IEEE International Conference on Robotics and Automation, 1996, pp. 1421-1426.

[4] R. Kurazume and S. Hirose, "Study on cooperative positioning system: optimum moving strategies for cps-iii," in IEEE International Conference on Robotics and Automation, 1998, pp. 2896-2903.

[5] I. Rekleitis, G. Dudek, and E. Milios, "Multi-robot cooperative localization: A study of trade-offs between efficiency and accuracy," in IEEE/RSJ International Conference on Intelligent Robots and Systems, 2002.

[6] Robert Grabowski, Luis E. Navarro-Serment, Christiaan, J. J. Paredis, and Pradeep K. Khosla, "Heterogeneous teams of modular robots for mapping and exploration," Autonomous Robots, vol. 8, no. 3, pp. 293-308, 2000.
[7] Y. Zhang, Mark Yim, L. Ackerson, D. Duff, and C. Eldershaw, "Stam: A system of tracking and mapping in real environments," IEEE Journal on Wireless Communication, vol. 11, no. 6, pp. 87-96, December 2004.

[8] M. Park, S. Chittan, A. Teichman, and M. Yim, "Automatic configuration recognition methods in modular robots," International Journal of Robotics Research, (In submission).

[9] Daniel Gomez-Ibanez, Ethan Stump, Ben Grocholsky, Vijay Kumar, and Camillo Taylor, "The robotics bus: A local communications bus for robots," in Proceedings of the Society of Photo-Optical Instrumentation Engineers, 2004.

[10] Mark Yim, Y. Zhang, K. Roufas, D. Duff, and C. Eldershaw, "Connecting and disconnecting for chain self-reconfiguration with polybot," IEEE/ASME Transactions on Mechatronics, Special Issue on SelfReconfigurable Robots, vol. 7, no. 4, pp. 431-441, December 2002. 